\title{
REGIOSPECIFIC SYNTHESIS AND RATIONALIZATION OF THE REGIODIVERGENCE SOME SPIROOXINDOLES AS EXPECTED ANTIOXIDANT AGENTS
}

\author{
SAMEH A. RIZK, NAGLAA F. MAHMOUD, NORELDIN A. ABDELSATTAR, MAI S. ALI*
}

Chemistry Department, Science Faculty, Ain Shams University, Cairo, Egypt 11566.

\begin{abstract}
:
The spirooxindolo pyrrolidine frameworks form core units of many naturally occurring molecules possess significant pharmacological activities. The [3+2] cycloaddition reactions of azomethine ylides has been realized through multi-component reaction (MCR) affording regiospecific spiroindoline-3, 2'-pyrrolidine derivatives. The reactivity of the spiroindoline (increase $E_{\text {номо }}$ value) has been depended upon electron repelling and attracting groups. The behavior of spiroindoline towards carbon electrophile and nitrogen nucleophile can be investigated. All compound structures are fully supported by spectroscopic data and elemental analysis. The antioxidant activity for these synthesized compounds could be expected that highly antioxidant activity was with compounds which proved to possess high HOMO values
\end{abstract}

Keywords: $\alpha$ - amino acids; isatins; azomethine ylides; 4-aryl-4-oxo-2-butenoic acids; indoline; pyrrolidine; spirocyclic; antioxidant agent; HOMO; LUMO.

\section{INTRODUCTION:}

The design of multicomponent reactions (MCRs) is an important field of research from the point of view of combinatorial chemistry. ${ }^{1}$ Multi-component reactions, relating the intrinsic formation of several bonds in one step, have verified to be an efficient and powerful tool for the rapid formation of complex heterocyclic compounds in recent years. ${ }^{2}$ The MCR strategy is a significant approach utilized by researchers worldwide to create several libraries of molecules of miscellaneous biological activities and has gained prominence in organic, medicinal and combinatorial chemistry. ${ }^{3}$ In most of the cases, a single product is obtained by reacting three or more different substrates in a well-defined mode through MCRs. ${ }^{4}$ These time-efficient reactions are environmentally benign and atom economic. MCRs are costeffective because the expensive purification processes as well as protection-deprotection steps are non-existent. ${ }^{5}$ Although the first MCR dates back to the Strecker synthesis ${ }^{6}$ of $\alpha$-amino acid in 1850, the MCR strategy has been successfully utilized in Hantzsch's synthesis of 1,4-dihydropyridines ${ }^{7}$ and Robinson $>\mathrm{s}$ synthesis of alkaloid tropinone. ${ }^{8}$ Consequently, rapid development has been observed in three- and four-component reactions. Potential biological activities and widespread synthetic utilities of spirocyclic compounds have led to their identification as a class of heterocyclic compounds, which has formed considerable interest in the pharmaceutical industry and in the diversified field of organic synthesis due to the steric strain linked with their quaternary carbon atom. ${ }^{9}$ Moreover, increased potentiality is generally observed when two or more different heterocyclic moieties exist in a single molecule. ${ }^{10}$ Isatin based spiro compounds or spirooxindoles are important center structures found in many natural alkaloids as well as synthetic pharmaceuticals. ${ }^{11}$ Spirooxindo line have free radical scavenging activity toward hydroxyl. It is critical to develop a pharmacological approach to combat oxidative stress which may reduce the risk of diseases and help in promoting healthy life. ${ }^{12}$

\section{RESULTS AND DISCUSSION:}

The multicomponent 1, 3-dipolar cycloaddition (MCDC) of azomethine ylides, generated in situ via decarboxylative condensation of isatins and $\alpha$-amino acids with olefinic and acetylenic dipolarophiles, represents a key approach for the regio-specific construction of a variety of spirooxindoles. Recently, this route has become significant in combinatorial chemistry due to its process simplicity, mild conditions, atomic economy and extension of the scope of substrates. The three-component condensation of equimolar amounts of isatins, sarcosine and 
4-aryl-4-oxo-2-butenoic acids in boiling aqueous methanol (1:3) afforded the spirooxindole1 in moderate to excellent yields. Spirooxindole1 was exclusively formed by the above method were characterized by ${ }^{1} \mathrm{H}$ NMR, and elemental analyses. The regiochemical outcome of the cycloaddition was unambiguously confirmed by ${ }^{1} \mathrm{H}$ NMR.
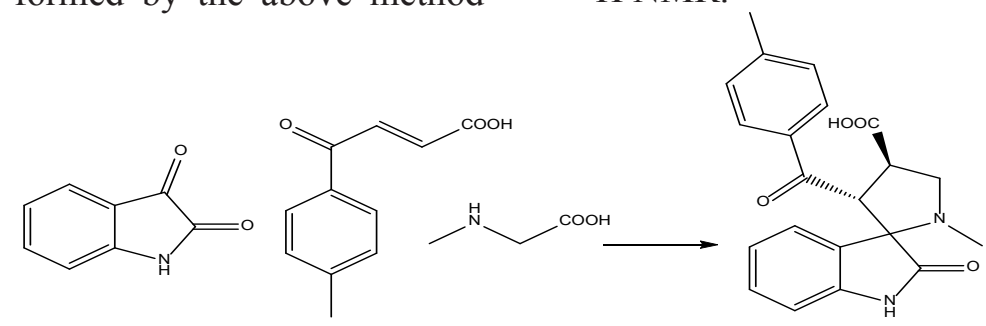

Scheme 1: Outline Regiospecific cycloaddition reaction of isatin, sacrosine, and aroylacrylic or chalcones via simple 1, 3-dipolar

In this reaction also two regioisomers can be expected, but in all above experiments solely the regioisomer1 is isolated without detectable trace amounts of other isomers. The higher reactivity of 4-aryl-4-oxo-2-butenoic acids ${ }^{13-17}$ induces remarkable rate acceleration and decreases the reaction time to only $10-15 \mathrm{~min}$ in a boiling mixture of methanol and water. The LUMO values of the aroyl acrylic acids are around ( $\mathrm{E}_{\text {LUMo }}$ $=-7.219$ ) that be lower than LUMO values of the chalcone derivatives $\left(\mathrm{E}_{\text {LUMO }}=-6.278\right)$. So, from point of view the cycloaddition reaction, the dipolarophile of the aroyl acrylic acids have been more reactive than the chalcone analogs. All compound structures are fully supported by spectroscopic data and elemental analysis as illustrated for compound $\mathbf{1}$. The ${ }^{1} \mathrm{H}$ NMR spectrum of compound 1 shows $3.01(2 \mathrm{H}, \mathrm{dd}, J=8.4$ $\left.\mathrm{Hz}, \mathrm{CH}_{2} \mathrm{~N}\right), 3.69(1 \mathrm{H}, \mathrm{q}, J=8.4 \mathrm{~Hz}, \mathrm{CHCOO})$, $4.43(1 \mathrm{H}, \mathrm{d}, J=11.4 \mathrm{~Hz}, \mathrm{CHCO})$, of pyrrolidine moiety show correlation signals to the neighboring methylene groups that was indicative for the assigned relative stereochemistry. The
$\mathrm{NH}$-proton of the oxindole moiety appeared as a singlet between 10.38-10.86 ppm. Interesting feature of this structure is a pincers-like conformation of the molecule. ${ }^{18,19}$ The stereochemistry of compound 1 was outlined the trans-configuration of the mentioned protons that be predeterminedby the trans-configuration of the initial aroyl acrylic acid, chalcone, maleic ester, endione, and dienone precursors. ${ }^{20,} 21$ The reaction between isatin and the $\alpha$-amino acid affords the azomethine ylide, which regioselectively adds to the $\mathrm{C}=\mathrm{C}$ bond of aroylacrylic acid or chalcone. The structures of the reacting systems, the azomethine ylide and dipolarophiles e.g. 4-chlorophenyl-4-oxo-2-butenoic acid and/or 4-arylidine imidazole-2,5-dione were investigated computationally. ${ }^{22}$ The geometrical structures of all possible conformers of the reacting systems were optimized using M06-2X. ${ }^{23}$ Since the stereochemistry of the cycloadduct1 was clarified by a single-crystal X-ray analysis, and the mechanism of the azomethine ylide formation by a decarboxylative route has been repeatedly described (Scheme 2). ${ }^{24-28}$

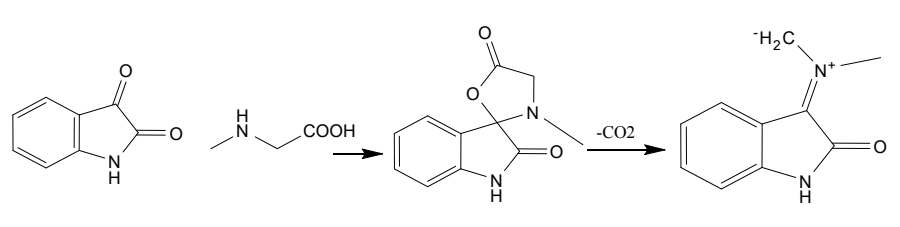

Scheme 2: Outline the mechanistic reaction of 1, 3-dipolar and dipolarophile.

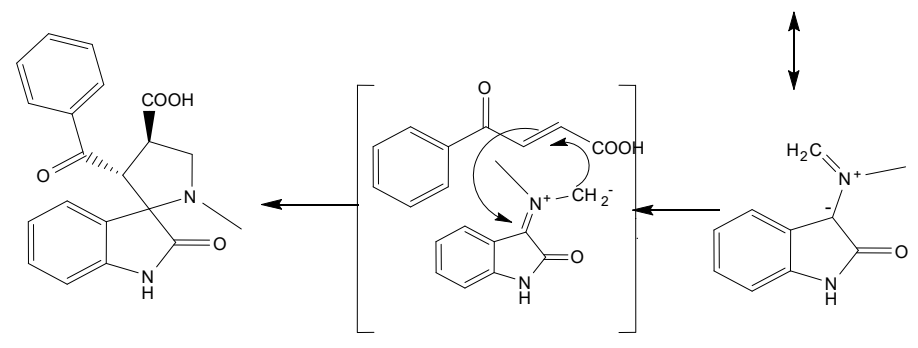


REGIOSPECIFIC SYNTHESIS AND RATIONALIZATION OF THE REGIODIVERGENCE 43

The presence of the electron donating groups directly attached for nitrogen ylide and electron withdrawing groups directly attached to carbon ylide were preceding the rate of the reaction due to increase the value of the HOMO energy.29The authors outline the effect of the electron repelling and attracting groups on the HOMO energy of the ylides. It will be assumed that HOMO and LUMO dipolarophile are affected by the same amount. The R3 groups in 1,3-dipolar precursor (Scheme 3) can be controlled not only in proceeding of the reaction due to further closes in the energy gap between HOMO1,3-dipolar and LUMOdipolarophile (Type 2) than the other HOMO-LUMO distance in case of sacrosine (Type 1) mak- ing this interaction clearly dominant. The authors wanted to prepare the new spirooxindoline derivatives have a high HOMO energy value to increase the efficiency as antioxidant reagents. This does not come only through the amendment of the installation of $\alpha$ amino acids. But the authors come across is regioselectivity in other words, the donating and withdrawing groups can be controlled in the value of EHOMO and molecular orbital coefficient in each 1,3-dipolar and dipolarophile. When the spiroindoline 1 was allowed to react with carbon electrophiles e.g. acetic anhydride, aspartic and docosanoic acids afforded N-substituted derivatives 2-4(Scheme 3).

Scheme 3: Reagents and conditions; (i) Acetic acid/POCl $/ 2 \mathrm{~h}$ on water bath, (ii) Aspartic acid/ $\mathrm{POCl}_{3} /$ reflux 2h, (iii) Docosanoic acid $/ \mathbf{P O C l}_{3} /$ reflux $2 \mathrm{~h}$.

On the other hands, the behavior of spiroindoline towards different nitrogen nucleophiles can be investigated. So, when the compound 1 was allowed to react with hydrazine hydrate, aniline,
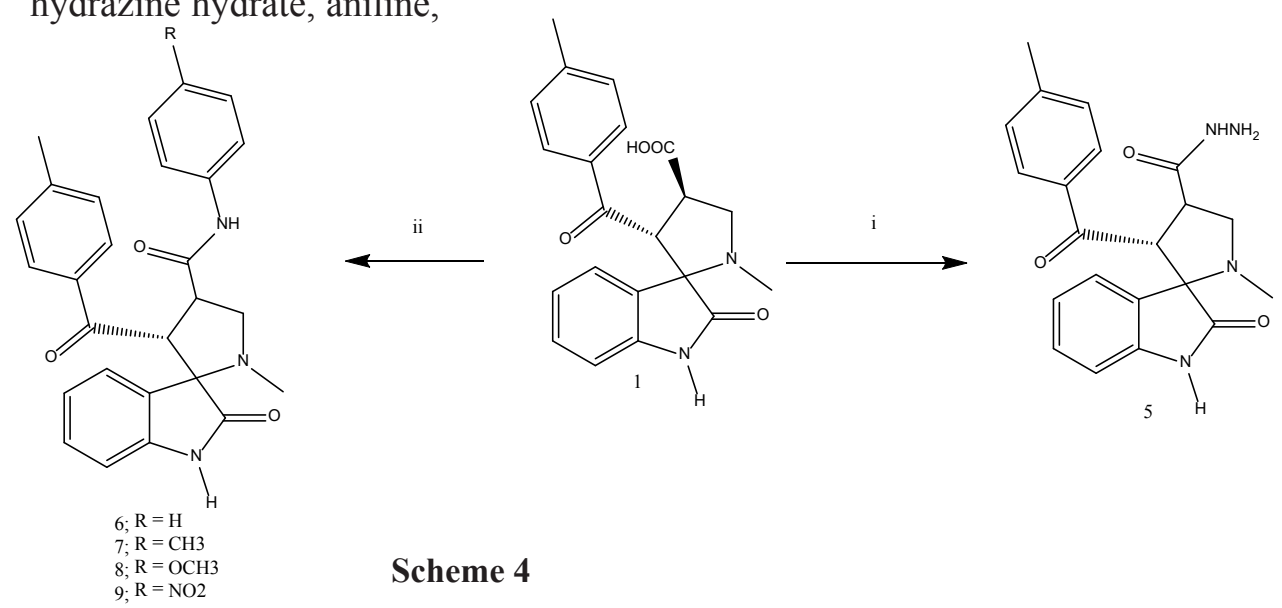

p-toluidine, $\mathrm{p}$-anisidine, and p-nitroaniline afforded hydrazide 5 and $\mathrm{N}$-aryl amide derivatives 6-9 (Scheme 4).
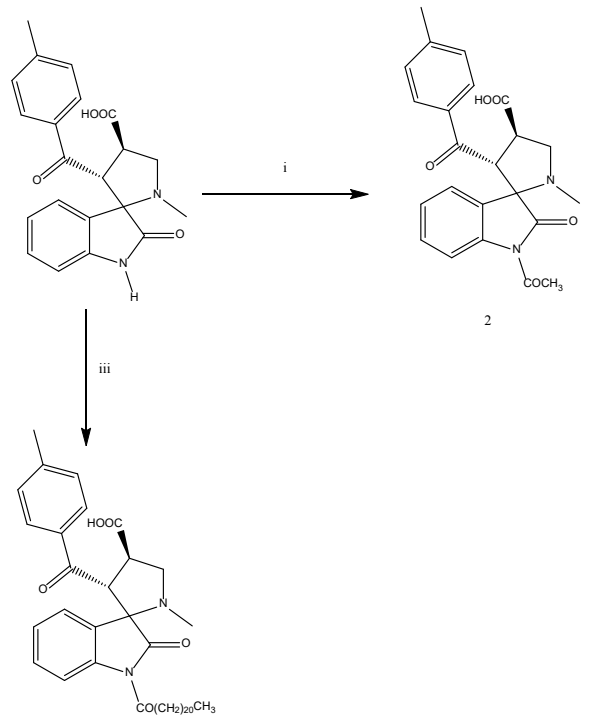
Reaction of the spiroindoline with benzene diazonium chloride didn't form azodye compound but it afforded N-phenyl derivative $\mathbf{1 0}$ via coupling with nitrogen of isatin i.e formation of

triaza compound followed by evolution of nitrogen. The reaction is considered aromatic substitution reaction. The product $\mathbf{1 0}$ was supported by elemental analysis. Scheme 5

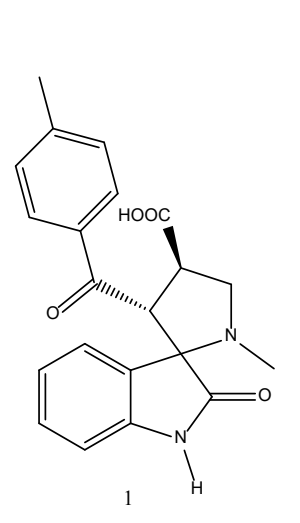

Experimental:

All melting points are uncorrected and determined on a Stuart Electric melting point apparatus (Microanalytical centre, Ainshams University, Cairo, Egypt). Elemental analyses were carried out by ElementarViro El-Microanalysis at the Micro-analytical Center, National Research Center, Egypt. IR spectra (KBr) were recorded on infrared spectrophotometer FT-IR 400D (New York, NY, USA) using OMNIC program and are reported frequency of absorption in terms of $\mathrm{cm}^{-1}$ and ${ }^{1} \mathrm{H}-\mathrm{NMR}$ spectra recorded on a Bruker spectrometer (Rheinstetten, Germany) at $400 \mathrm{MHz}$ using TMS as internal standard and with residual signals of the deuterated solvent $\delta=7.26 \mathrm{ppm}$ for $\mathrm{CDCl}_{3}$ and $\delta 2.51 \mathrm{ppm}$ for DMSO-d6. ${ }^{13} \mathrm{C}-\mathrm{NMR}$ spectra were recorded on the same spectrometer (Rheinstetten, Germany) at $100 \mathrm{MHz}$ and referenced to solvent signals $\delta$ $=77 \mathrm{ppm}$ for $\mathrm{CDCl}_{3}$ and $\delta 39.50 \mathrm{ppm}$ for DMSO- $d_{6}$. DEPT 135 NMR spectroscopy was used where appropriate to aid the assignment of signals in the ${ }^{1} \mathrm{H}$ - and ${ }^{13} \mathrm{C}$-NMR spectra. The mass spectra were recorded on Shimadzu GCMSQP-1000 EX mass spectrometer (Kyoto, Japan) used the electron ionization technique at $70 \mathrm{e.v}$. Homogeneity of all synthesized compounds was checked by TLC .

Typical procedure for the synthesis of spirooxindoline derivatives from the threecomponent reaction of isatin, sarcosine and
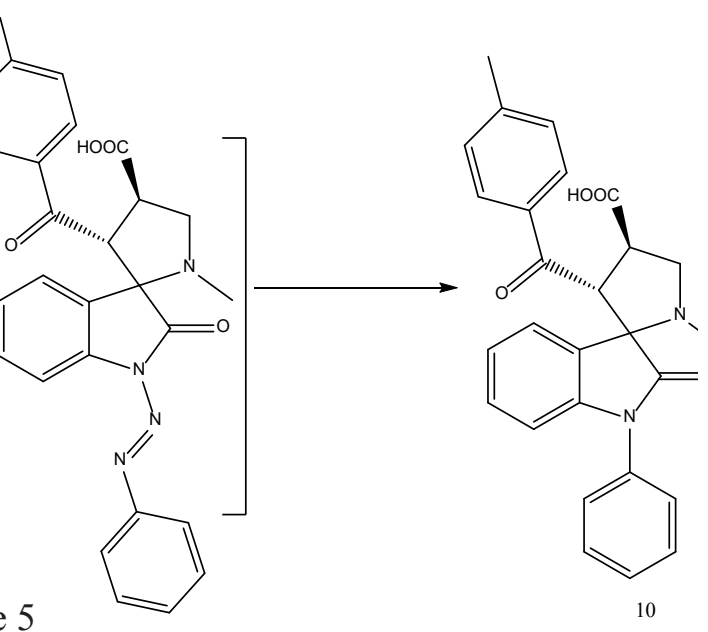

10

4-(4-methylphenyl)-4-oxo-2-butenoic acid .

(3'R, 4'R)-1'-methyl-3'-(4methylbenzoyl)-2-oxospiro[indoline-3,2' pyrrolidine]-4'-carboxylic acid(1) A mixture of isatin(1.1 g; $0.01 \mathrm{~mol}), \operatorname{sarcosine}(0.9 \mathrm{~g}$; $0.01 \mathrm{~mol})$ and 4-(4-methylphenyl)-4-oxo-2butenoic acid(1.6g; $0.01 \mathrm{~mol})$ in $10.0 \mathrm{~mL}$ aqueous methanol $(1: 3)$ was heated in an oil bath to reflux for $1 \mathrm{~h}$ or stirred at room temperature. The resulting precipitate was collected by filtration, washed with cold methanol and recrystallized from benzene.

Characterization of the synthesized compounds

(Compounds 2-4) . A mixture of compound $\mathbf{1}$ (3.64 g, $0.01 \mathrm{mmol})$ and the acid e.g. acetic acid, aspartic acid and/or decosanoic acid $(0.01 \mathrm{mmol})$ in $\mathrm{POCl}_{3}(15 \mathrm{ml})$ was heated for $2 \mathrm{~h}$. The solvent was evaporated under vacuum, and then iced water $(25 \mathrm{~mL})$ was added. The solid obtained was filtered off and recrystallized from proper solvent.

(3'R, 4'R)-1-acetyl-1'-methyl-3'-(4methylbenzoyl)-2-oxospiro [indoline-3, 2'-pyrrolidine]-4'-carboxylic acid (2). Brown crystals(dioxane), $65 \%, \mathrm{mp}>300^{\circ} \mathrm{C}$; IR $\left(v_{\text {max }}\right.$, $\left.\mathrm{cm}^{-1}\right)$ : 1617-1734 (CO), ${ }^{1} \mathrm{H}$ NMR (DMSO- $d_{6}$ ), $\delta$, ppm: 12.19(1H, s, COOH), 2.31-2.62-2.56 $\left(9 \mathrm{H}, \mathrm{s}, 3\left(\mathrm{CH}_{3}\right)\right)$, 6.70-7.64 (8H, m, ArH), 4.192.95 (1H, dd,2-CH(pyrolidine)), 2.61-2.55 $\left(2 \mathrm{H}, 2 \mathrm{dd}, \mathrm{CH}_{2}\right.$ (pyrolidine)); Anal. Calcd for 
$\mathrm{C}_{23} \mathrm{H}_{22} \mathrm{~N}_{2} \mathrm{O}_{5}(406): \mathrm{C}, 67.97 ; \mathrm{H}, 5.46 ; \mathrm{N}, 6.89 \%$. Found: C, 67.49; $\mathrm{H}, 5.31 ; \mathrm{N}, 6.90 \%$.

$(3, R, 4, R)-1$ - ( 3 - a m i n o - 3 carboxypropanoyl) -1 '- methyl-3'-(4methylbenzoyl)-2-oxospiro[indoline-3,2'pyrrolidine]-4'-carboxylic acid(3).Dark brown crystals (benzene), $72 \%, \mathrm{mp} 82-84^{\circ} \mathrm{C}$; IR $\left(v_{\max }\right.$, $\left.\mathrm{cm}^{-1}\right): 1675(\mathrm{CO}),{ }^{1} \mathrm{H}$ NMR (DMSO- $\left.d_{6}\right), \delta, \mathrm{ppm}$ : $13.89(1 \mathrm{H}, \quad \mathrm{s}, \mathrm{COOH}), 2.41-2.26-3.56(3 \mathrm{H}$, $\left.\mathrm{S}, \mathrm{CH}_{3}\right), 6.75-7.68(8 \mathrm{H}, \mathrm{m}, \mathrm{ArH}), 4.39-3.29(1 \mathrm{H}$, dd, $\mathrm{CH}$ (pyrrolidine)), 2.71-2.455 (2H, 2dd, $\mathrm{CH}_{2}$ (pyrrolidine) $), 1.50\left(2 \mathrm{H}, \mathrm{d}, \mathrm{NH}_{2} \mathrm{D}_{2} \mathrm{O}\right.$ exchangeable), $3.8(1 \mathrm{H}, \mathrm{m}, \mathrm{CH}), \quad 2.85-2.595\left(2 \mathrm{H}, \mathrm{d}, \mathrm{CH}_{2}\right)$; Anal. Calcd for $\mathrm{C}_{26} \mathrm{H}_{29} \mathrm{~N}_{3} \mathrm{O}_{7}(495): \mathrm{C}, 63.02 ; \mathrm{H}$, $5.90 ; \mathrm{N}, 8.48 \%$. Found: C, 62.92; H, 5.40; N, $8.52 \%$.

(3'R,4'R)-1-docosanoyl-1'-methyl-3'-(4methylbenzoyl)-2-oxospiro[indoline-3,2'pyrrolidine]-4'-carboxylic acid(4) .Buff crystals(toluene), $75 \%$, mp $112-114^{\circ} \mathrm{C}$; IR ( $v_{\max }$, $\left.\mathrm{cm}^{-1}\right): 1732,1716,1697,1650$ (CO), ${ }^{1} \mathrm{H}$ NMR $\left(\mathrm{DMSO}-d_{6}\right), \delta$, ppm: $12.43(1 \mathrm{H}, \mathrm{s}, \mathrm{COOH}), 2.51-$ $2.46\left(9 \mathrm{H}, \mathrm{s}, 3\left(\mathrm{CH}_{3}\right)\right), 6.75-7.68(8 \mathrm{H}, \mathrm{m}, \mathrm{ArH})$, 4.09-2.99 (1H, dd,CH(pyrrolidine)), 2.71-2.46 $\left(2 \mathrm{H}, 2 \mathrm{dd}, \mathrm{CH}_{2}\right.$ (pyrrolidine)), 1.26-2.39 $(40 \mathrm{H}$, m,20( $\left.\mathrm{CH}_{2}\right)$ ).; Anal. Calcd for $\mathrm{C}_{43} \mathrm{H}_{62} \mathrm{~N}_{2} \mathrm{O}_{5}(686)$ : C, 75.18; H, 9.10; N, 4.08 \%. Found: C, 75.57; H, 9.43; N, 4.25\%.

(Compound 5 -9) .A mixture of compound $1(3.64 \mathrm{~g}, 0.01 \mathrm{mmol})$, hydrazine hydrate or aromatic and amines $(0.01 \mathrm{mmol})$ in ethanol $(30 \mathrm{~mL})$ was refluxed for $3 \mathrm{~h}$. The solvent was evaporated under vacuum. The solid obtained was filtered off and recrystallized from proper solvent.

(3'R)-1'-methyl-3'-(4-methylbenzoyl)2-oxospiro[indoline-3,2'-pyrrolidine]-4'carbohydrazide (5) . Green crystals (toluene), $82 \%$. mp $220-222^{\circ} \mathrm{C}$; IR $\left(v_{\max }, \mathrm{cm}^{-1}\right): 3143$ (NH), 1699,1613 (CO), ${ }_{1}^{1} \mathrm{H}$ NMR (DMSO- $d_{6}$ ), $\delta, \quad$ ppm:,2.41-2.26-2.56(6H,s, $\left.2\left(\mathrm{CH}_{3}\right)\right), 6.75-$ $7.68(8 \mathrm{H}, \quad \mathrm{m}, \quad \mathrm{ArH}), 4.09-2.99 \quad(1 \mathrm{H}, \mathrm{dd}$, $\mathrm{CH}$ (pyrrolidine) $), 2.71-2.455(2 \mathrm{H}, \quad 2 \mathrm{dd}$, $\mathrm{CH}_{2}$ (pyrrolidine) $), \quad 10.56\left(1 \mathrm{H}, \mathrm{s}, \mathrm{NH} \quad \mathrm{D}_{2} \mathrm{O} \quad\right.$ exchangeable), $9.08\left(1 \mathrm{H}, \mathrm{t}, \mathrm{NH} \mathrm{D}_{2} \mathrm{O}\right.$ exchangeable), 4.22(2H, d, $\mathrm{NH}_{2} \mathrm{D}_{2} \mathrm{O}$ exchangeable);Anal. Calcd for $\mathrm{C}_{21} \mathrm{H}_{22} \mathrm{~N}_{4} \mathrm{O}_{3}(378): \mathrm{C}, 66.65 ; \mathrm{H}, 5.86 ; \mathrm{N}$,
14.81\%. Found: C, 66.32; H, 6.305; N, 14.53\%.

(3'R) -1'-methyl-3'-(4-methylbenzoyl)2-oxo-N-phenylspiro [indoline-3, 2'-pyrrolidine]-4'- carboxamide (6). Pale yellow crystals (toluene) , 70\%. mp $218-220^{\circ} \mathrm{C}$; IR $\left(v_{\max }, \mathrm{cm}^{-1}\right): 3014(\mathrm{NH}), 1700,1614(\mathrm{CO}) .{ }^{1} \mathrm{H}$ NMR (DMSO- $\left.d_{6}\right), \delta, \quad$ ppm:,2.41-2.26-2.56 $\left(6 \mathrm{H}, \mathrm{s}, 2\left(\mathrm{CH}_{3}\right)\right), 6.75-7.68(8 \mathrm{H}, \mathrm{m}, \mathrm{ArH}), 2.81-$ 2.56(1H, dd, $\mathrm{CH}$ (pyrrolidine) ), 2.71-2.455(2H, $2 \mathrm{dd}, \mathrm{CH}_{2}$ (pyrrolidine) $), 10.66\left(1 \mathrm{H}, \mathrm{s}, \mathrm{NH} \mathrm{D}_{2} \mathrm{O}\right.$ exchangeable); Anal. Calcd for $\mathrm{C}_{27} \mathrm{H}_{25} \mathrm{~N}_{3} \mathrm{O}_{3}$ (439): C, 73.78; H, 5.73; N, 9.56\%. Found: C, 73.50; $\mathrm{H}, 5.51 ; \mathrm{N}, 9.25 \%$.

(3'R)-1'-methyl-3'-(4-methylbenzoyl)2-oxo-N-(p-tolyl) spiro [indoline-3, 2'-pyrrolidine]-4'-carboxamide (7) . Buff crystals (toluene), $72 \%$ mp $212-214^{\circ} \mathrm{C}$; IR $\left(v_{\max }, \mathrm{cm}^{-1}\right): 3337(\mathrm{NH}), 1695,1613(\mathrm{CO}),{ }^{1} \mathrm{H}$ NMR $\quad\left(\mathrm{DMSO}-d_{6}\right), \quad \delta, \quad$ ppm,:,2.41-2.322.26(9H,s,3( $\left.\left.\mathrm{CH}_{3}\right)\right), 6.75-7.68$ (8H, m, $\left.\mathrm{ArH}\right)$, 2.81-2.56(1H, dd, CH(pyrrolidine)), 2.71-2.455 $\left(2 \mathrm{H}, 2 \mathrm{dd}, \mathrm{CH}_{2}\right.$ (pyrrolidine)), $10.66(1 \mathrm{H}, \mathrm{s}, \mathrm{NH}$ $\mathrm{D}_{2} \mathrm{O}$ exchangeable); Anal. Calcd for $\mathrm{C}_{28} \mathrm{H}_{27} \mathrm{~N}_{3} \mathrm{O}_{3}$ (453): C, 74.15; H, 6.00; N, 9.27\%. Found: C, $74.61 ; \mathrm{H}, 5.53 ; \mathrm{N}, 8.19 \%$.

(3'R)-N-(4-methoxyphenyl)-1'-methyl3'-(4-methylbenzoyl)-2-oxospiro [indoline-3, 2'-pyrrolidine]-4'-carboxamide (8). Black crystals (toluene), $65 \%$ mp $224-226^{\circ} \mathrm{C}$; IR $\left(v_{\max }, \mathrm{cm}^{-1}\right): 3423(\mathrm{NH}), 1660(\mathrm{CO}),{ }^{1} \mathrm{H}$ NMR $\left(\mathrm{DMSO}-d_{6}\right), \quad \delta, \quad$ ppm:, 2.41-2.81 $(1 \mathrm{H}, \mathrm{dd}$, $\mathrm{CH}$ (pyrrolidine)), $2.71-2.455\left(2 \mathrm{H}, 2 \mathrm{dd}, \mathrm{CH}_{2}\right.$ (pyrr olidine)), 3.81-2.26-2.56 (3H,s, $\left.\mathrm{CH}_{3}\right), 6.75-7.45$ $(8 \mathrm{H}, \mathrm{m}, \mathrm{ArH}), 10.05-10.66\left(2 \mathrm{H}, \mathrm{s}, 2 \mathrm{NH} \mathrm{D}_{2} \mathrm{O}\right.$ exchangeable);; Anal. Calcd for $\mathrm{C}_{28} \mathrm{H}_{27} \mathrm{~N}_{3} \mathrm{O}_{4}(469)$ : C, 71.62 H, 5.80; N, 8.95\%. Found: C, 71.17; H, $6.34 ; \mathrm{N}, 8.87 \%$.

(3'R)-1'-methyl-3'-(4-methylbenzoyl)$\mathrm{N}$-(4-nitrophenyl)-2-oxospiro [indoline-3, 2'-pyrrolidine]-4'-carboxamide (9) . Gray crystals (benzene), $45 \%$. mp $84-88^{\circ} \mathrm{C}$; IR $\left(v_{\max }, \mathrm{cm}^{-1}\right): 3431,3302(\mathrm{NH}), 1624(\mathrm{CO}) .{ }^{1} \mathrm{H}$ NMR $\left(\mathrm{DMSO}-d_{6}\right), \quad \delta, \quad$ ppm:,2.41-2.26-2.56 $\left(3 \mathrm{H}, \mathrm{S}, \mathrm{CH}_{3}\right), 6.75-8.17(8 \mathrm{H}, \mathrm{m}, \mathrm{ArH}), 2.81-$ $2.56(1 \mathrm{H}, \mathrm{dd}, \mathrm{CH}$ (pyrrolidine)),2.71-2.455 $\left(2 \mathrm{H}, 2 \mathrm{dd}, \mathrm{CH}_{2}\right.$ (pyrrolidine) $), 10.05-10.66(2 \mathrm{H}$, $\mathrm{s}, 2 \mathrm{NH} \mathrm{D}_{2} \mathrm{O}$ exchangeable); Anal. Calcd for 
$\mathrm{C}_{27} \mathrm{H}_{24} \mathrm{~N}_{4} \mathrm{O}_{5}$ (484): C, 66.93; H, 4.99; N, 11.56\%. Found: C, 66.66; H, 5.48; N, 11.75\%.

(3'R,4'R)-1'-methyl-3'-(4-methylbenzoyl)2 - oxo-1 - ph e ny ls piro [i ndoline-3,2' pyrrolidine]-4'-carboxylic acid(10). A mixture of compound $1(3.64 \mathrm{~g}, 0.01 \mathrm{mmol})$, aniline $(0.93 \mathrm{~g}, 0.01 \mathrm{mmol}), \mathrm{NaNO}_{2}$ solution and $\mathrm{HCl}$ was stirred for $1 \mathrm{~h}$. The solid obtained was filtered off and recrystallized from ethanol. Brown yellow crystals, 70\%, mp104 - 106 ${ }^{\circ} \mathrm{C}$; IR $\left(v_{\max }, \mathrm{cm}^{-1}\right): 1791,1592(\mathrm{CO}) .{ }^{1} \mathrm{H}$ NMR $\left(\mathrm{DMSO}-d_{6}\right), \delta, \mathrm{ppm}:, 12.18(1 \mathrm{H}, \mathrm{s}, \mathrm{COOH})$, 2.41-2.26-2.56(6H,s, $\left.2\left(\mathrm{CH}_{3}\right)\right), \quad 6.75-7.55(8 \mathrm{H}$, $\mathrm{m}, \mathrm{ArH}), 4.09-2.99$ (1H,dd, $\mathrm{CH}$ (pyrrolidine)), 2.71-2.455 (2H,2dd, $\mathrm{CH}_{2}$ (pyrrolidine)), Anal. Calcd for $\mathrm{C}_{27} \mathrm{H}_{24} \mathrm{~N}_{2} \mathrm{O}_{4}(440)$ : C, 73.62; $\mathrm{H}, 5.49$; N, 6.36\%. Found: C, 73.98; H, 5.72; N, 6.36\%.

\section{REFERENCES:}

(a) WeberL., Illegen K., AlmstetterM., Synlett, (1999), 366 ; (b) ArmstrongR. W., CombsA. P., TempestP. A., Brown S. D. KeatingT. A., Acc. Chem. Res., (1996), 29, 123.

(a) JiangB., Regale T.,Wever W., S.-J. Tu and G. Li, Chem. -Asian J., (2010), 5, 2318 ; (b) Maiti S., Biswas S. and Jana U., J. Org. Chem., (2010), 75, 1674 ; (c)Tietze L. F.,Brasche G. andGericke K. M., Domino Reactions in Organic Synthesis, Wiley-VCH, Weinhein,(2006); (d) de Meijere A.,von Zezschwitz P. andBrase S., Acc. Chem. Res., (2005), 38, 413 , and references cited therein.

(a)Bienayme H.,Hulme C.,Oddon G.,Schmitt P., Chem. Eur. J., (2000), 6, 3321-3329 ; (b) Balme G.,Bossharth E.,Monteiro N., Eur. J. Org. Chem.,( 2003), 41014111 ; (c) Brase S.,Gil C.,Knepper K., Bioorg. Med. Chem., (2002), 10, 2415-2437; (d) Domling A. and Ugi I., Angew. Chem., Int. Ed., (2000), 39, 3168-3210.

(a)Tempest P. A., Curr. Opin. Drug Discovery Dev., (2005), 8, 776 ; $\quad$ (b)Fujioka H.,Murai K.,Kubo O.,Ohba Y.,Kita Y., Org. Lett.,(2007), 9, 1687 ; (c)Evdokimov N. M.,Kireev A. S., Yakovenko A. A.,Antipin M. Y.,Magedov I. V.,Kornienko A., J. Org. Chem.,(2007), 72, 3443 ; (d)Wang X. S., Li Q.,Wu J. R., Li Y. L., Yao C. S.,Tu S. J., Synthesis, (2008), 1902 .

(a)Trost B. M., Angew. Chem., Int. Ed., (1995), 34, 259 ; (b)Wender P. A., Handy S. T.,Wright D. L., Chem. Ind., (1997), 765

Strecker A., Liebigs Ann. Chem.,(1850), 75, 27 .

Hantzsch A., Justus Liebigs Ann. Chem.,(1882), 215, 1 .

Robinson R., J. Chem. Soc.,(1917), 111, 876.
Hilton S. T.,Ho T. C. T., Pljevalijcic G.,Jones K., Org. Lett.,(2000), 17, 2639 .

Balini R.,Bosica G., Conforti M., Magi R., Mazzacannt A.,Righi P.,Sarton G., Tetrahedron, (2001), 57, 1395 .

(a)Kang T. H., Matsumoto K.,Murakami Y.,Takayama H., Kitajima M., Aimi N.,Watanabe H., Eur. J. Pharmacol., (2002), 444, 39 ; (b)Edmondson S., Danishefsky S.,Sepp-lorenzinol L.,Rosen N., J. Am. Chem. Soc.,(1999), 121, 2147.

Sultana, R.; Butterfield, D.A. Neurochem. Res. (2004), 29, 2215-2220.

El-Hashash, M.A.; Rizk, S.A. J. Heterocycl. Chem.(2015), $52,1248-1252$.

El-Hashash, M.; Rizk, S.; Aburzeza, M. Egypt J. Chem. (2011), 54, 383-391.

Rizk, S.; El-Hashash, M.; Shaker, S; Mostafa, K. Egy. J. Chem.(2012), 55, 1-13.

EL-Hashash, M.A.; Rizk, S.A. Op. J. Org. Chem.(2013), $3,27-32$.

Rizk, S.; El-Hashash, M.; Mostafa, K. Egy. J. Chem. (2008), 51, 116-123.

Tatyana, L.P.; Fedor, G.Y.; Victoria, V.L.; Svetlana, V.S.; Oleg, V.S.; Vladimir, I.M.; Alexander, S.K. Beilstein J Org Chem.(2014), 10,117-126.

Bin, Y.; Xiao-Nan, S.; Xiao-Jing, S.; Ping-Ping, Q.; YiChao, Z.; De-Quan, Y.; Hong-Min, L. Steroids(2015), $102,92-100$.

Yong-Mei, X.; Yu-Qin, Y.; Hong-Bao, S.; Ting-Ting, Y.; Jie, L.; Tai-Ran, K. Molecules(2011), 16, 8745-8757.

Jun, H.; Guang, O.; Zhixiang, Y.; Rongsheng, T.; Jianyou, S.; Liang, O. A Molecules(2013), 18, 5142-5154.

Lashgari, N.; Ziarani G. Arkivoc(2012), i, 277-320.

Zhao, Y.; Truhlar, D. Theor Chem Acc.(2008), 120, 215 241.

George, R.F.; Ismail, N.S.; Stawinski, J.; Girgis, A.S. Eur. J Med Chem. (2013), 48, 339-351.

Girgis, A.S.; Stawinski, J.; Ismail, N.S.; Farag, H. Eur. J Med Chem.((2012), 47, 312-22.

Kia, Y.; Osman, H.; Suresh Kumar, R.; Murugaiyah, V.; Basiri, A.; Khaw, K.Y.; Rosli, M.M. J. Med Chem. (2014), $10,512-520$.

Kia, Y.; Osman, H.; Kumar, R.S.; Murugaiyah, V.; Basiri, A.; Perumal, S.; Razak, I.A. Bioorg. Med. Chem. Lett. (2013), 23, 2979-2983.

Almansour, A.I.; Kumar, R.S.; Arumugam, N.; Basiri, A.; Kia, Y.; Ali, M.A.; Farooq, M.; Murugaiyah, V. Molecules(2015), 20, 2296-2309.

Liu, J.T.; Lin, W.W.; Jang, J.J.; Liu, J.Y.; Yan, M.C.; Hung, C.; Kao, D.H.; Wang, Y.; Yao, C.F. Tetrahedron.(1999), $55,7115-7128$. 